GU J Sci, Part C, 6(4): 938-946 (2018)

Gazi Üniversitesi
Fen Bilimleri Dergisi
PART C: TASARIM VE TEKNOLOJI
http://dergipark.gov.tr/gujsc

\title{
Ultra Geniş Band Uygulamaları için Düzlemsel Hat Beslemeli Mikroşerit Anten Tasarımı
}

\author{
Mehmet Ali BELEN ${ }^{1, *}$ \\ ${ }^{I}$ Artvin Çoruh Üniversitesi, Mühendislik Fakültesi, Elektrik Elektronik Mühendisliği Bölümü, Seyitler/ARTVIN
}

\begin{abstract}
Öz
Makale Bilgisi

Başvuru: 11/07/2018

Kabul: $19 / 10 / 2018$

Anahtar Kelimeler

Mikroșerit Anten

Ultra Geniş Band

Eş Düzlemsel Hat Besleme

Keywords

Haberleşme alanındaki geniş bant gereksinimlerden ötürü geniş bantlı antenler günümüzde popüler hale gelmiştir. Son yıllarda gerek askeri gerekse ticari uygulamalarda boyutları küçültülmüs ancak aynı zamanda geniș bant genişliğine sahip antenlere olan ihtiyaç artmaktadır. $\mathrm{Bu}$ bağlamda, kompakt, hafif, küçük boyut, düşük maliyet ve geniş bant genişliği gibi kavramlar kablosuz iletişim sistemleri tasarımcıları tarafından gerçekleştirilmesi gereken önemli zorlukların başında gelmektedir. Bu bağlamda mikroşerit antenler, basit yapıları, küçük boyutları, düzgün ve eğimli yüzeylere uygulanabilmeleri ve sert yüzeylere uygulandığında dayanıklı olmaları gibi birçok dikkat çekici özelliği ile öne çıkmaktadır. Bu çalışma kapsamında ultra geniş band uygulamaları için $3 \mathrm{GHz}$ ile $11 \mathrm{GHz}$ arasında çalışan eş düzlemsel hat besleme (CPW-fed) yapısına sahip mikroşerit anten tasarımı yapılmıştır. Tasarımlarda CST Microwave Studio programı kullanılmıştır. Anten tasarımında $1.56 \mathrm{~mm}$ kalınlıklı FR4 ve 4.4 epsilon değerinde taban üzerine tasarlanmıştır. Anten $24 \mathrm{mmx} 40 \mathrm{~mm}$ boyutlarındadır. Yapılan benzetimlerde band genişliği, $S_{11}$, kazanç ve 1şıma örüntüsü incelenmiş, parametrelerde değişiklikler yapılarak en iyi performansı gösteren anten yapısı belirlenmiş ve üretimi yapılarak ölçümleri tamamlanmıştır. Yapılan ölçümler sonucunda üretilen anten modelinin $3 \mathrm{GHz}$ ile $11 \mathrm{GHz}$ arasında geridönüş kaybı $-\mathrm{S}_{11}<-10 \mathrm{~dB}$, kazancı ise $1 \mathrm{~dB}$ ile $3.7 \mathrm{~dB}$ arasında elde edilmiştir
\end{abstract}

Microstrip Antenna

Ultra-Wideband

Coplanar wavequide

feeding

\section{Design of Coplanar Wavequide Feeding Microstrip Antenna Design for Ultra-Wideband Applications}

\begin{abstract}
Due to the broadband requirements in the field of communication, broadband antennas are becoming popular nowadays. In recent years, both military and commercial applications have been reduced in size, but at the same time the need for broadband antennas is growing. In this context, concepts such as compactness, light weight, small size, low cost and wide bandwidth are the major challenges that wireless communication system designers have to face. In this context, microstrip antennas stand out with their remarkable features such as their simple construction, their small size, their ability to be applied to smooth and sloping surfaces, and their durability when applied to hard surfaces. In this study, a microstrip antenna design with coplanar line feed (CPW-fed) structure operating between $3 \mathrm{GHz}$ and $11 \mathrm{GHz}$ was applied for ultrawide band applications. CST Microwave Studio program was used in the designs. The antenna design is based on the Fr4 base of $1.56 \mathrm{~mm}$ thickness and 4.4 epsilon. The antenna is 24 $\mathrm{mm} \times 40 \mathrm{~mm}$. Bandwidth, $\mathrm{S}_{11}$, gain and radiation pattern were investigated in the simulations made, the antenna structure showing the best performance was determined by making changes in the parameters and the measurements were completed by the production. As a result of the measurements made, the gain of the antenna model between $3 \mathrm{GHz}$ and $11 \mathrm{GHz}$ is $\mathrm{S}_{11}<-10 \mathrm{~dB}$ and the gain is between $1 \mathrm{~dB}$ and $3.7 \mathrm{~dB}$.
\end{abstract}




\section{GİRIŞ (INTRODUCTION)}

Son dönemlerde ultra geniş band (UWB) haberleşme teknolojisi araştırmacıların ilgisini çekmeye başlamıştır. 3.1-10.6GHz lik band genişliği amerika birleşik devletleri ulusal haberleşme komisyonu'nun (FCC) tarafından ultra geniş bandlı sistemlerin kullanımı için tahsis edilmiştir. Böylece UWB teknolojisine olan ilgi artmıştır. UWB antenler, geniş frekans aralıklarında çalışmalarından dolayı, çok kanallı veya çok sistemli uygulamalarda kullanım kolaylığı sağlamaktadır. Bu nedenle UWB anten tasarımına bir dönem yoğun bir ilgi olmuştur[1]. Düzenleme kuruluşlarının frekans bantlarına getirdiği kısıtlamalar, farklı yöntemlerdeki teknolojik gelişmeler vb. durumlar UWB anten tasarımına olan ilgiyi azaltmıştır. Ancak son yıllarda amerikan düzenleme kuruluşu, Federal Communication Commission (FCC)'in görüntüleme, algılama ve haberleşme gibi yoğun ilgili gören uygulamalar için UWB bant aralığındaki geliştirme çalışmalarına verdiği izinler sayesinde UWB anten tasarımına ilgi yeniden artmıştır. FCC tarafından açıklanan UWB tanımı, yansıma kaybının $\mathrm{S}_{11}(\mathrm{~dB})<-10 \mathrm{~dB}$ olduğu frekans bant genişliğinin, merkez frekansa oranının $\% 25$ den fazla olması durumudur[2]. Bu çalışmada UWB uygulamaları için FCC tarafından lisanssız kullanıma açılan frekans bandı olan 3-11GHz frekans aralığı kullanılmıştır.

Kablosuz iletişim insan hayatında son yıllarda daha da önem kazanmaktadır. Dolayısı ile kablosuz iletişim askeri savunma, sağlık sistemleri, acil çağrı sistemleri gibi farklı alanlarda da kullanılmaktadır[35]. Bu nedenle son yıllarda UWB çalışmalarında monopol düzlemsel mikroşerit antenler üzerindeki çalışmalar hız kazanmıştır. Monopol antenlerin en büyük avantajları düzlemsel bir yapıya sahip olmaları ve çok büyük band genişliklerine sahip olmalarıdır. Mikroşerit anten ile ilgili literatürde yer alan çalışmaların büyük bir kısmı, dikdörtgen, daire ve üçgen gibi, düzgün geometriler üzerinde yoğunlaşmıştır [6-9]. Bu geometriler şekil 1'de verilmiştir.
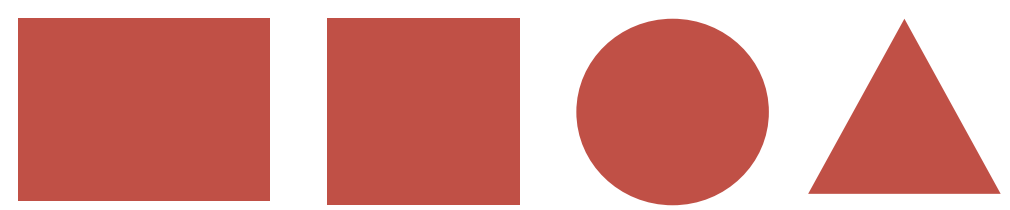

Şekil 1. Mikroşerit anten geometrileri.

Düzlemsel anten geometrilerinde besleme modeli olarak eş düzlemsel hat besleme (CPW-fed) yapıs1 yaygın olarak kullanılmaktadır[10],[11]. Bu çalışmada 3-11GHz ultra geniş band frekanslarıyla uyumlu, düşük maliyetli ve boyutlu, S11<-10dB seviyesi altında, kazanc1 1.4-4dB arasında değişen monopol özelliğe sahip mikroşerit anten tasarımı ve üretimi yapılmıştır. Tasarımlarda CST Microwave Studio programı kullanılmıştır. Yapılan benzetimlerde band genişliği, $S_{11}$, kazanç ve 1şıma örüntüsü incelenmiş, parametrik analiz yapılarak hatların etkisi incelenmiştir böylelikle en iyi performansı gösteren anten geometrisi belirlendi ve üretimi yapılarak ölçümleri alınmıştır.

\section{ANTEN TASARIMI (ANTENNA DESIGN)}

Mikroşerit anten tasarımında; yama boyutları (Işıma alanı), taban kalınlığı (h), yama ve toprak düzlemi kalınlığı (t) ve dielektrik sabiti $(\mathcal{E} r)$ anten performansını etkileyen temel özelliklerdir. Çalışma kapsamında ilk aşamada anten tasarımının önemli parametreleri incelenmiştir. Bu parametreler; geri dönüş kaybı, yönlendiricilik, kazanç, polarizasyon, duran dalga oranı başlıkları altında toplanmıştır. Anten tasarımında $1.56 \mathrm{~mm}$ kalınlıklı Fr4 ve 4.4 epsilon değerinde taban üzerine tasarlanmıştır. Anten parametreleri tablo 1'de verilmiştir. UWB anten modelinin benzetimleri CST-MWS yazılımı kullanılarak yapılmıştır. Anten geometrik modeli şekil 2'de verilmiştir. Eş-düzlemsel hat besleme yapısına sahip antenin toprak düzlemi ile 1şıma yaması aynı düzlem üzerinde bulunmaktadır. Eş düzlemsel hat besleme ile entegrasyonu kolay olmakta ve az kayıplı olacaktır. 


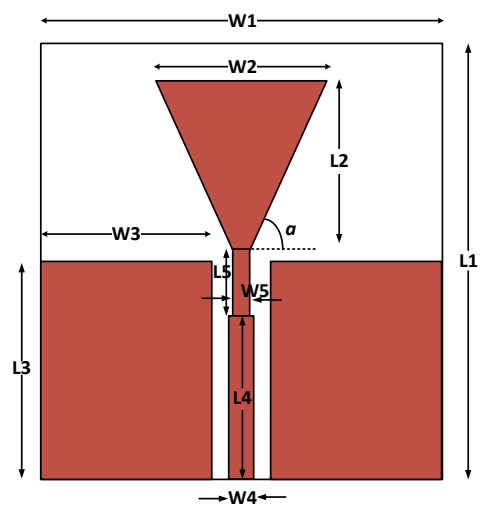

Şekil 2. Anten geometrisi.

Tablo 1. Anten Tasarım Değişkenleri

\begin{tabular}{|l|l|l|l|}
\hline W1 & 23.43 & L1 & 39.77 \\
\hline W2 & 18.8 & L2 & 14.77 \\
\hline W3 & 9.36 & L3 & 15 \\
\hline W4 & 1.92 & L4 & 12 \\
\hline W5 & 1.59 & L5 & 3 \\
\hline Malzeme, $\mathrm{cr} / \mathrm{H}$ & FR4, 4.4/ $1.58 \mathrm{~mm}$ & a / gap & $600 / 1.2 \mathrm{~mm}$ \\
\hline
\end{tabular}

Şekil 3'de tasarlanan birim anten modelinin $\mathrm{S}_{11}$, frekans boyunca anten kazancının değişimi ve anten verimi verilmiş̧ir. Çalışma frekansı $3 \mathrm{GHz}$ ile $11 \mathrm{GHz}$ arasında $\mathrm{S}_{11}$ değeri $-10 \mathrm{~dB}$ altında, kazanc1 $1.5 \mathrm{~dB}$ ile $4 \mathrm{~dB}$ arasında ve toplam verimliliği \%80 ile \%96 arasında değişmektedir.

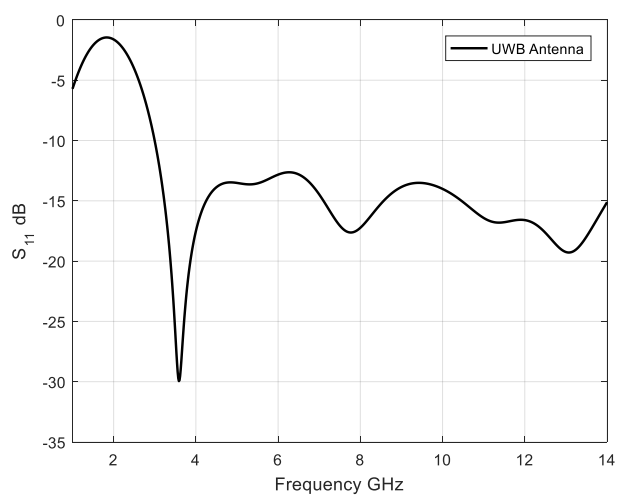

(a)

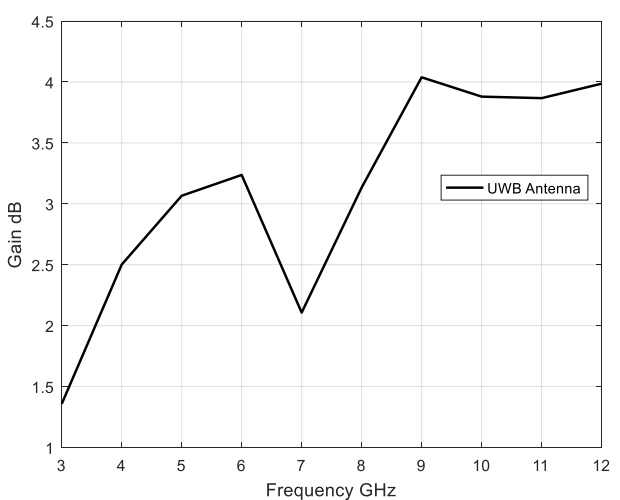

(b)

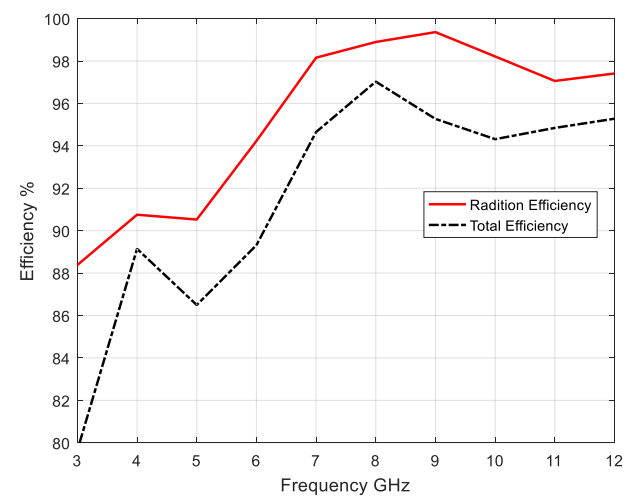

(c)

Şekil 3. (a) Geridönüş Kaybı $S_{11}$ (b) Frekans boyunca anten kazancı (c) Verimlilik. 
Şekil 4'te tasarlanan antenin 3boyutlu ışıma örüntüsü verilmiştir. Işıma şekillerinden görüleceği üzere anten 1şıması monopol özelliğe sahiptir. Frekans arttıkça anten 1şıması üst kısma doğru toplanmaya başlamıştır.

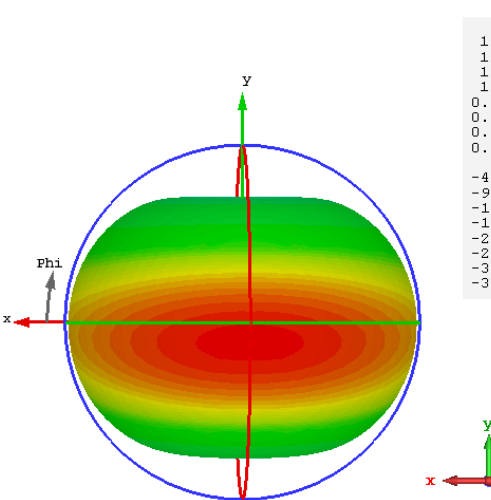

(a)

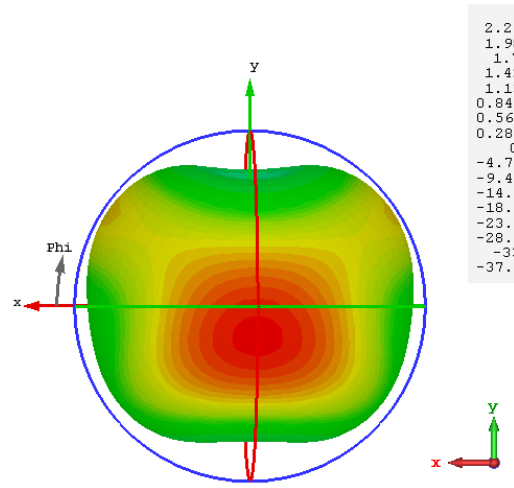

(c)

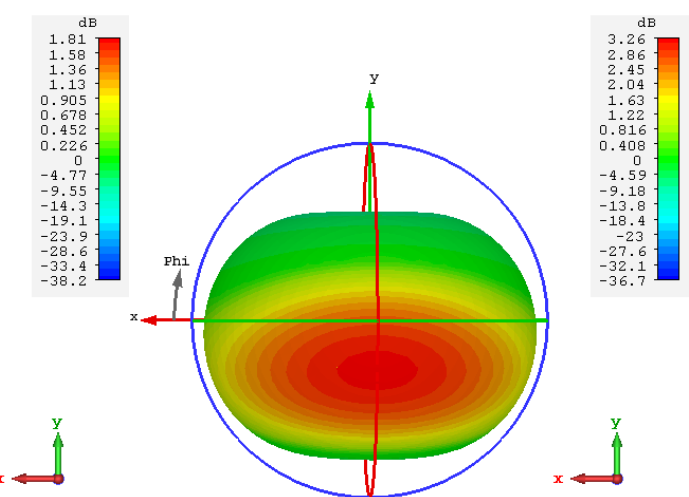

(b)

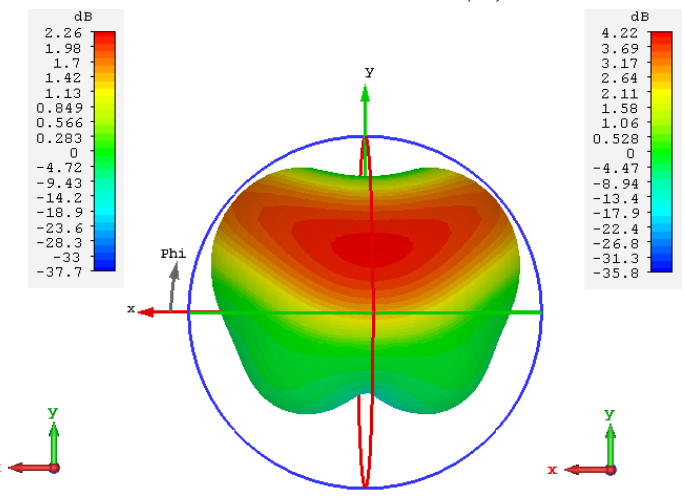

(d)

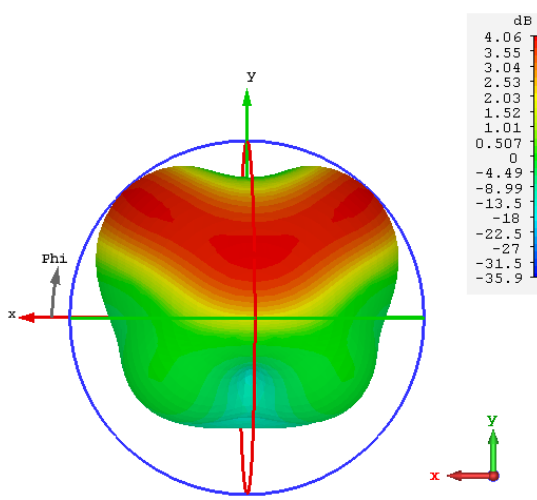

(e)

Şekil 4. Işıma Örüntüsü (a) $3 \mathrm{GHz}$ (b) $5 \mathrm{GHz}$ (c) $7 \mathrm{GHz}$ (d) $9 \mathrm{GHz}$ (e) $10 \mathrm{GHz}$.

\section{PARAMETRÍK İNCELEME ( PARAMETRIC ANALYSIS )}

Çalışma kapsamında tablo 1'de yer alan anten parametrelerinden " $a$ ", "gap", " $\mathrm{L}_{2}$ ", "L $\mathrm{L}_{5}$ " değişkenlerinin rezonans frekansı ve frekans boyunca kazancı incelenmiștir. Şekil 5'te " $a$ " açı değerinin değişiminin anten performansına etkisi incelenmiştir. " $a$ " değişken değeri $52^{0}$ ile $66^{0}$ arasında incelenmiştir. $52^{0}$ değerince $\mathrm{S}_{11}$ değeri $4 \mathrm{GHz}$ ile $8 \mathrm{Ghz}$ arasında $-15 \mathrm{~dB}$ seviyelerinde iken, $66^{\circ}$ değerince -10 seviyesine çıkmıştır. $5 \mathrm{GHz}$ ile $8 \mathrm{Ghz}$ arasında $52^{0}$ ile $66^{\circ}$ arasında yaklaşık $1 \mathrm{~dB}$ kazanç farkı vardır. $8-10 \mathrm{GHz}$ arasında ise $60^{\circ}$ çalışma bandında ise kazancı ve $S_{11}$ değeri kullanışlı bulunmuştur. 


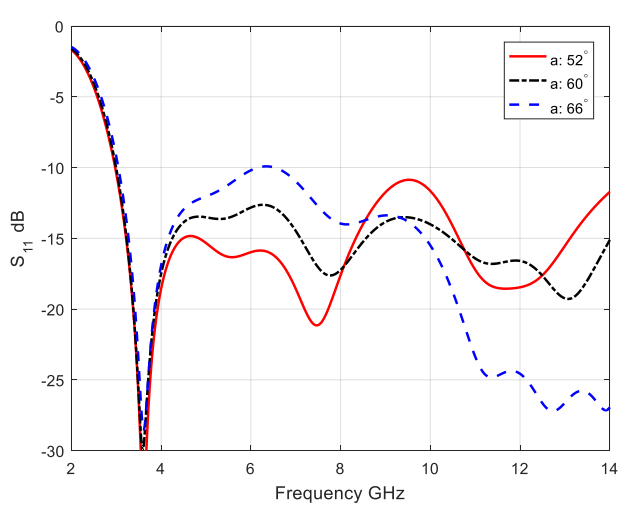

(a)

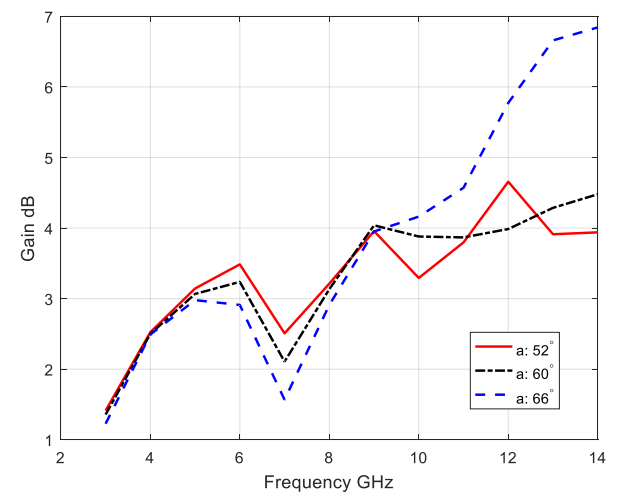

(b)

Şekil 5. (a) Geridönüş kaybı $S_{11}$ (b) Frekans boyunca anten kazancı.

Şekil 6'da "gap" eş-düzlemsel hat beslemesinin boşluk miktarının değişimi incelenmiştir. "gap" değerinin değişiminin antenin rezonans frekansında 300MHzlik bir kayma oluşturmaktadır. Kazanç değişiminde ise çalışma frekansında benzer eğriler elde edilmektedir.

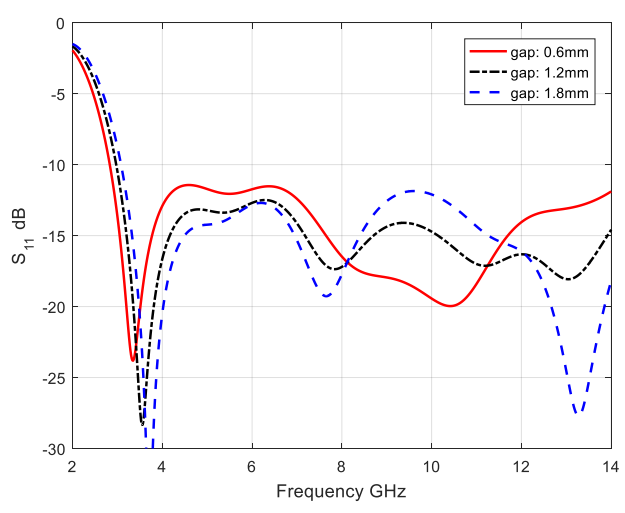

(a)

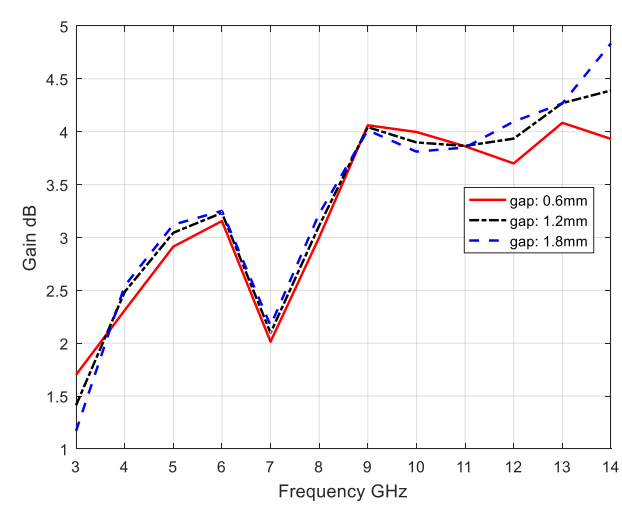

(b)

Şekil 6. (a) Geridönüş kaybı $S_{11}$ (b) Frekans boyunca anten kazancı.

Şekil 7'de " $\mathrm{L}_{2}$ " anten uzunluğu incelenmiştir. Anten uzunluğu arttıkça rezonans frekansı alt frekanslara inmekte ve anten boyutu arttı̆ğndan kazancıda artmaktadır.

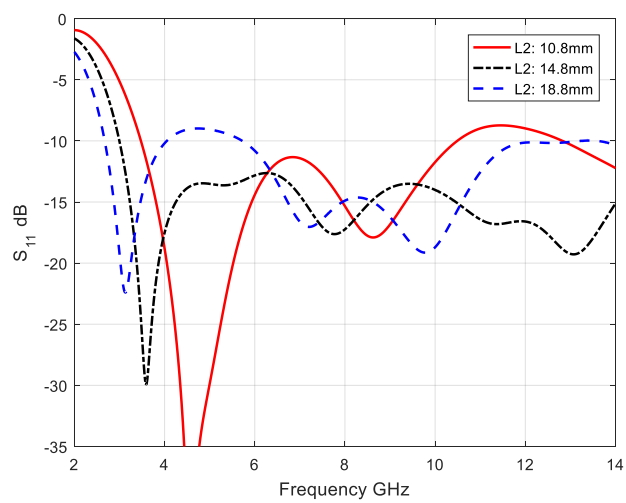

(a)

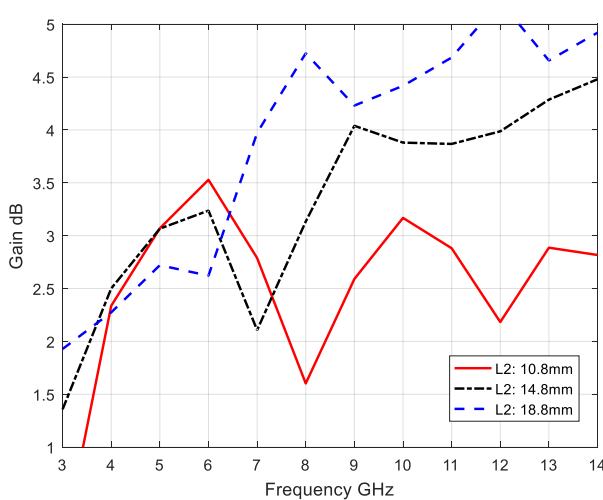

(b)

Şekil 7. (a) Geridönüş kaybı $S_{11}$ (b) Frekans boyunca anten kazancı

Şekil 8'de "L," besleme hattının uyumlandırma kısmının uzunluğu incelenmiştir. Uyumlandırma hat uzunluğu arttıkça rezonans frekansı aşağıya inmekte ve anten boyutu arttığından kazancıda artmaktadır. 


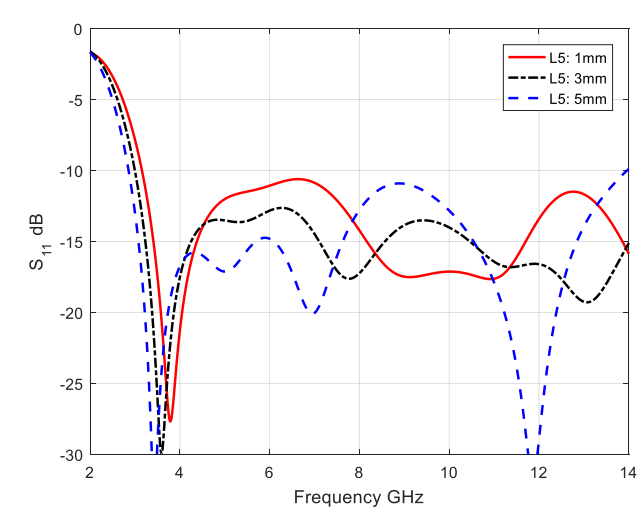

(a)

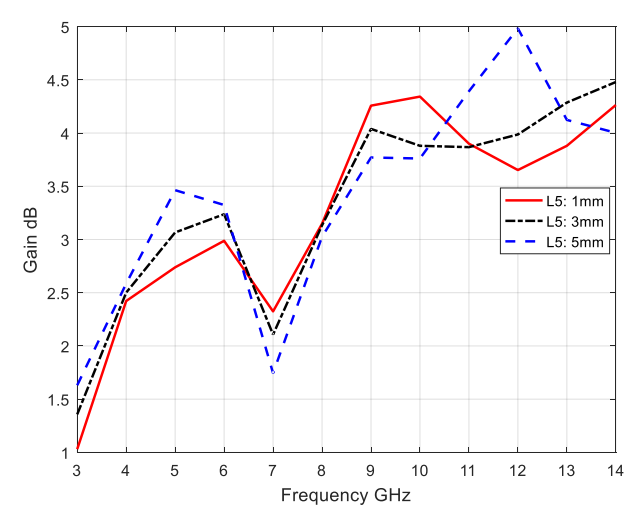

(b)

Şekil 8. (a) Geridönüş kaybı $S_{11}$ (b) Frekans boyunca anten kazancı

Antenin parametrik incelemesi sonucunda $\mathrm{L}_{2}$ ve $\mathrm{L}_{5}$ değişkenlerinin değiştirilmesi ile anten boyu değişeceğinden anten rezonans frekansının değiştiği gözlenmiştir. Eş-düzlemsel hat beslemesinin boşluk miktarının değişimi (gap) ile anten kazancı değişmediği ancak başlangıç rezonans frekansının değiştiği gözlenmiştir. " $a$ " açı değerinin değişiminin anten performansına etkisi incelenmiştir. " $a$ " $52^{0}$ ile $66^{0}$ arasında incelenmiştir. $5 \mathrm{GHz}$ ile $8 \mathrm{Ghz}$ arasında $52^{\circ}$ ile $66^{\circ}$ arasında yaklaşık $1 \mathrm{~dB}$ kazanç farkı vardır. 8$11 \mathrm{GHz}$ arasında ise $60^{\circ}$ çalışma bandında ise kazancı ve $\mathrm{S}_{11}$ değeri kullanışlı bulunmuştur. Yapılan tasarımda, geri dönüş kaybı, kazanç, verimlilik ve band genişliği arasındaki denge sağlanmıştır.

\section{4. ÜRETIM ve ÖLÇÜM ( FABRICATION and MEASUREMENT )}

Anten tasarımındaki önemli parametreler (antenin giriş empedansı, etkin yüzeyi, duran dalga oranı, 1şıma gücü, polarizasyon, 1şıma örüntüsü, kazancı, huzme genişliği) incelenmiştir. Tasarımlar ve simülasyonlar aracılığıyla mikroşerit anten parametreleri ve besleme şekilleri incelenmiştir. Yapılan parametrik incelemeler sonucunda $3 \mathrm{GHz}$ ile $11 \mathrm{GHz}$ ultra geniş band frekanslarında uyumlu, düşük boyutlu, $\mathrm{S}_{11}<-10$, monopole özellikte, $1.5 \mathrm{~dB}$ ile $4 \mathrm{~dB}$ kazanç özelliklerine sahip tablo 1 deki anten geometrisinin üretimi yapılmıştır. Üretilen anten modeli şekil 9'da verilmiştir.

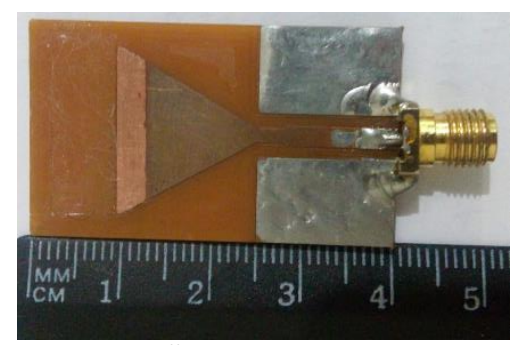

Şekil 9. Üretilen anten modeli.

Üretilen modülünün (şekil 11) geri yansıma katsayısı $\left(S_{11}\right)$, ve 1şıma paterni şekil 10'daki ölçüm düzeneği kullanılarak ölçülmüştür. Ölçümlerde referans anten olarak $0.8-18 \mathrm{GHz}$ arasında çalışan dual ridged horn anten kullanılmıştır[12].

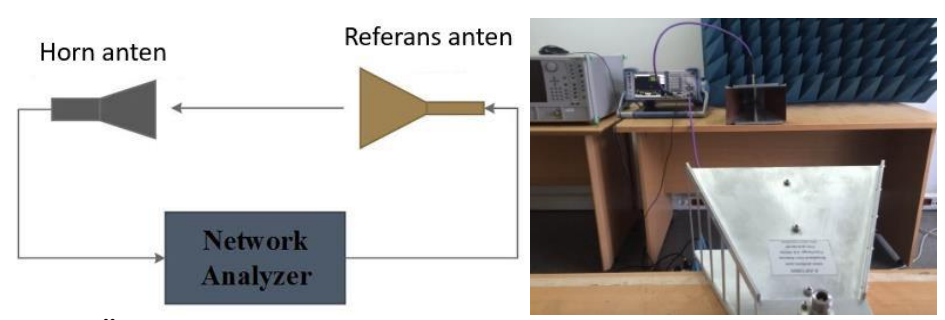

Şekil 10. Ölçüm düzeneği ve laboratuar ortamında ölçüm kurulumu.

Şekil 11'de üretilen antenin geri dönüş kaybı ölçülmüştür. 3.1-10.6GHz rezonans frekansında $\mathrm{S}_{11}$ değeri $15 \mathrm{~dB}$ altında ölçülmüştür. 


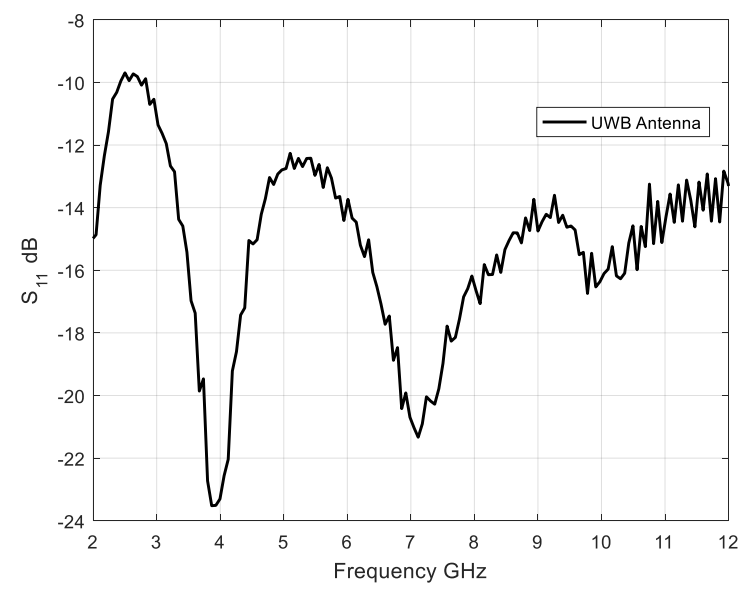

Şekil 11. Geri dönüş kaybı $\left(S_{11}\right)$ ölçüm sonucu.

Tablo 2'de üretimi yapılan antenin şekil 10'daki ölçüm düzeneği kullanılarak yapılan ölçüm ve benzetim sonuçları verilmiştir. Yapılan ölçümlerde çalışma bandı boyunca anten kazancı 1dB ile $3.7 \mathrm{~dB}$ arasında değiştiği gözlenmiştir.

Tablo 2. Simulasyon ve ölçüm sonuçlarının karşılaştırılması

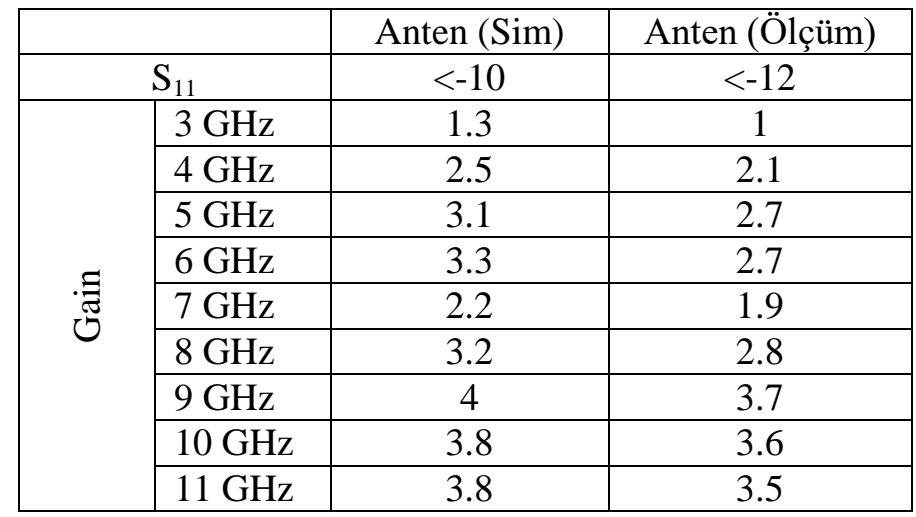

Tablo 3'te üretimi yapılan anten modeli ile literatürdeki modeller ile kıyaslaması yapılmıştır. 3-11GHz frekans bandını kapsamaktadır. $\mathrm{S}_{11}$ değeri $-12 \mathrm{~dB}$ 'nin altındadır. Anten kazancı çalışma frekansı boyunca $1 \mathrm{~dB}$ ile $3.7 \mathrm{~dB}$ arasında değişmektedir. Anten maliyetini düşürmek ve temini kolay olması sebebiyle üretimde FR4 malzeme kullanılmıştır. Boyut olarak diğerlerinden daha az alan kaplamaktadır.

Tablo 3. Anten Performansı Literatür Karşılaş̧ırması

\begin{tabular}{|l|c|c|c|c|c|}
\hline & Bu çalışma & {$[\mathbf{1 3}]$} & {$[\mathbf{1 4}]$} & {$[\mathbf{1 5}]$} & {$[\mathbf{1 6}]$} \\
\hline $\begin{array}{l}\text { Çalışma } \\
\text { Bandı (GHz) }\end{array}$ & $3-11$ & $3.3-11$ & $3.8-11$ & $3.28-12.44$ & $2.2-10$ \\
\hline $\mathbf{S}_{\mathbf{1 1}}(\mathbf{d B})$ & $-12<$ & $-10<$ & $-10<$ & $-6<$ & $-6<$ \\
\hline Kazanç (dB) & $1-3.7$ & --- & --- & $3 \mathrm{~dB} @ 5.2 \mathrm{GHz}$ & $5.6 \mathrm{~dB} @ 6 \mathrm{GHz}$ \\
\hline Boyut (mm) & $24 \times 40 \times 1.56$ & $36 \times 43 \times 0.79$ & $30 \times 40 \times 0.79$ & $30 \times 39 \times 1.6$ & $45 \times 31 \times 1.27$ \\
\hline Malzeme & FR4 & Arlon AD270 & RT/Duroid 5870 & FR4 & Rogers 5880 LZ \\
\hline
\end{tabular}

\section{SONUÇ (CONCLUSION)}

Geniş band antenler son yıllarda haberleşme alanındaki ihtiyaçlardan dolayı popüler hale gelmiştir. $\mathrm{Bu}$ bağlamda ticari ve askeri uygulamalar için küçük boyutlu, hafif, geniş bandlı anten tasarımları önem kazanmıştır. Bu çalışma kapsamında ultra geniş band uygulamaları için $3 \mathrm{GHz}$ ile $11 \mathrm{GHz}$ arasında çalışan eş düzlemsel hat besleme (CPW-fed) yapısına sahip mikroşerit anten tasarımı yapılmıştır. Tasarımlar ve anten performans parametreleri CST-MWS yazılımında yapılan benzetimler ile elde edilmiştir. Eşdüzlemsel hat besleme yapısına sahip antende toprak düzlemi ile 1şıma yaması aynı düzlem üzerinde 
bulunmaktadır. Anten $24 \mathrm{mmx} 40 \mathrm{~mm}$ boyutlarındadır. Antenin parametrik incelemesi sonucunda $\mathrm{L}_{2}$ ve $\mathrm{L}_{5}$ değişkenlerinin değiştirilmesi ile anten boyu değiş̧eceğinden anten rezonans frekansının değiştiği gözlenmiştir. Eşdüzlemsel hat beslemesinin boşluk miktarının değişimi (gap) ile anten kazancı değişmediği ancak başlangıç rezonans frekansının değiştiği gözlenmiştir. " $a$ " açı değerinin değişiminin anten performansına etkisi $52^{\circ}$ ile $66^{\circ}$ arasında incelenmiştir. $5 \mathrm{GHz}$ ile $8 \mathrm{Ghz}$ arasında $52^{\circ}$ ile $66^{\circ}$ arasında yaklaşı $1 \mathrm{~dB}$ kazanç farkı vardır. $8-10 \mathrm{GHz}$ arasında ise $60^{\circ}$ çalışma bandında ise kazancı ve $\mathrm{S}_{11}$ değeri kullanışlı bulunmuştur. Yapılan tasarımda, geri dönüş kaybı, kazanç ve band genişliği arasındaki denge sağlanmıştır. Yapılan ölçümler sonucunda üretilen anten modelinin $3 \mathrm{GHz}$ ile $11 \mathrm{GHz}$ arasında geridönüş kayb1 - $\mathrm{S}_{11}<-12 \mathrm{~dB}$, kazancı ise $1 \mathrm{~dB}$ ile 3,7 $\mathrm{dB}$ arasında elde edilmiştir.

\section{TEŞEKKÜR (ACKNOWLEDGMENTS)}

Bu çalışma “2017.F14.02.01” nolu proje Artvin Çoruh Üniversitesi Bilimsel Araştırma Projeleri Destekleme Programı kapsamında desteklenmektedir.

\section{KAYNAKLAR (REFERENCES)}

[1] J. L. Volakis, Antenna Engineering Handbook, Fourth Edition, McGrawHill., New York, 2007.

[2] FCC 02-48, 2002. Commission's Rules Regarding Ultra-Wideband Transmission Systems, FCC, 98153 , Washington

[3] G. Şahan, F. Erden, E Başaran, E, Düşük Arka/Yakın Yan Kulakçık Seviyesine Sahip Dizi Beslemeli Parabolik Reflektör Anten Tasarımı. Gazi Üniversitesi Fen Bilimleri Dergisi Part C: Tasarım ve Teknoloji, 5 (3), 1-12. (2017). Retrieved from http://dergipark.gov.tr/gujsc/issue/31140/338316

[4] S. Kulaç. Medikal İmplant Haberleşme Sistemleri İçin Bandgenişliği Verimli Örtüşmeli Fsk Kodlamalı Güvenli Komut İletimi. Gazi Üniversitesi Fen Bilimleri Dergisi Part C: Tasarım ve Teknoloji, 6 (2), 250-258, 2018. DOI: 10.29109/http-gujsc-gazi-edu-tr.288622

[5] A. Apak, İ. Üstoğlu. Türkiye İçin Yeni Nesil Acil Çağrı Sistemi. Gazi Üniversitesi Fen Bilimleri Dergisi Part C: Tasarım ve Teknoloji, 5 (3), 127-137, 2017. DOI: 10.29109/http-gujsc-gazi-edutr.338344

[6] C. A. Balanis, Antenna Theory: Analysis and Design, Wiley Interscience, 1136, 2005.

[7] J. R. James, P. S. Hall, Handbook of Microstrip Antennas, Peter Peregrinus Ltd., London, 1312, 1989.

[8] R. Garg, P. Bhartia, I. Bahl, A. Ittipiboon, Microstrip Antenna Design Handbook, Artech House, London, 875, 2001.

[9] G. Kumar, K.P. Ray, Broadband Microstrip Antennas, Artech House, London, 409, 2003

[10] E. Garduno-Nolasco, Comparison between quasi circular planar antennas with microstrip and CPW feeding, LATINCOM 2010 IEEE, Latin American, pp. 1-5, 2010.

[11] M. Moktaari, J. Bornemann, Directional Ultra Wideband Antennas in Planar Technologies, Microwave Conference, EuMC 2008. 38th European, pp. 885-888, 2008.

[12] A-info, lb8180, 0.8-18 Ghz broad band horn antenna available at: http://www.ainfoinc.com/en/p_ant_h_brd.asp

[13] L. Xiaochi, H. Junguang, Z. Qingchun, J. Zhaoneng, M. Chao, A Novel Flower-shaped Microstrip Antenna for UWB Communication, International Applied Computational Electromagnetics Society Symposium (ACES), Suzhou, China, 2017 
[14]M. Mohammadirad, N. Komjani, M. Yazdi, Design and Implementation of a New UWB Microstrip Antenna, 14th International Symposium on Antenna Technology and Applied Electromagnetics \& the American Electromagnetics Conference, Ottawa, ON, Canada, 2010

[15] D.D. Ahire, G.K. Kharate, Corner Rounded UWB Monopole Rectangular Microstrip Patch Antenna", IEEE Applied Electromagnetics Conference (AEMC), Aurangabad, India, 2017

[16] M.I. Hussein, E. Serria, Metamaterial Effect on UWB Circular Microstrip Antenna, International Conference on Electrical and Computing Technologies and Applications (ICECTA), Ras Al Khaimah, United Arab Emirates, 2017 